\title{
A NOVEL INDEX FOR TEMPORAL STABILITY ANALYSIS IN SPACE AND TIME OF SAR-DERIVED SCENES
}

\author{
M. E. Molinari *, A. Monti-Guarnieri, M. Manzoni
}

Politecnico di Milano - Dipartimento di Elettronica, Informazione e Bioingegneria (DEIB), Via Ponzio 34/5, 20133 Milan, Italy (moniaelisa.molinari, andrea.montiguarnieri, marco.manzoni)@polimi.it

\section{KEY WORDS: SAR, Change Detection, Coherence, Temporal Stability Index, Dunes, Sand Mitigation}

ABSTRACT: Detecting temporal changes is one of the most important applications of satellite sensors. In recent years, the increasing availability of regular time-series of SAR imagery, provided by the Sentinel-1 mission of the European Space Agency (ESA), has drawn increasing attention to these techniques, especially in earth environment monitoring and risk management. Within this paper, a coherent change detection analysis for evaluating the risk due to movements of dunes and sand sheets in desertic areas is proposed. To this purpose, we introduce a novel, coherence-based index, named Temporal Stability Index (TSI), that is suited for characterizing the percentage of stability of a target with time. TSI maps can be generated over areas as wide as hundreds of kilometers, in a short time, and mostly by exploiting available software tools (plus some simple coding). The information provided is complementary to the average of the short-term coherence, here shown. Results of analysis performed on two desertic regions (the United Arab Emirates and Egypt) document the usefulness of TSI for the identification of dune movements and areas subject to sand accumulation, supporting risk mitigation measures.

\section{INTRODUCTION}

Change detection represents one of the most relevant applications of remote sensing and it is applied to a broad range of environmental monitoring applications, e.g. change detection over time of forests, water, wetland, and ice (Rignot, van Zyl, 1993; Engeset et al., 2002; Preiss et al., 2006; Dabboor et al., 2015; Durieux et al., 2019), or damage assessment caused by flooding, volcanic ash, earthquake, etc. (Tamura, 2015; Jung et al., 2016; Jung et al., 2017; Monti-Guarnieri et al., 2018).

Within this context, SAR (Synthetic Aperture Radar) observations have a privileged role since, compared to optical observations, they are not affected by time or weather conditions, such as different sunlight illumination and/or cloud cover. Furthermore, in recent years, the place into the orbit of the Sentinel-1 satellites constellation of the European Space Agency (ESA) gave a significant boost to research by making systematically, ubiquitous and freely available regular time series of SAR data, with a revisiting time capability up to 1-3 days.

The detection of changes based on SAR observations involves the comparison of a pair of coregistered images acquired over the same area at two different times. For the detection process, both coherent and incoherent methods can be used.

Incoherent change detection techniques are based on the mean backscatter intensity, including polarimetric observations; this makes them comparable to optical images methods. Incoherent techniques sense changes mainly due to humidity and surface roughness due to vegetation, wind over water, man-made structures.

The coherent change detection methods take advantage of Interferometric SAR (InSAR) images, by exploiting both phases and amplitudes to identify the changes. Most of them are based on the estimation of the coherence (Ferretti et al., 2007) between SAR image pairs. As coherence is the linear correlation coefficient, its absolute value, normalized in $[0,1]$, is highly sensitive to the stability of the scene.
The joint exploitation of amplitude and phase makes coherence sensitive to changes in the speckle - that is subcentimetric scene changes in the reflectivity - that may remain undetected using only intensity information. This is the reason why the coherent technique is largely preferred for most change detection applications.

The work here presented exploits the coherent techniques to identify instabilities in desertic areas due to dunes and sand sheets movements, which represent a significant threat to transportation, urban areas, and population. Within this context, characterized by continuous targets movement over time, the computation of coherence seems not to be able to provide a comprehensive knowledge of the phenomenon under study; the planning of measures for dune movement risk mitigation requires added valuable information about the behavior of the phenomenon over time, e.g. how much time a scene remains stable.

To fulfill this requirement, we introduce a novel, coherencebased index, aimed to perform an efficient and robust analysis of the temporal variation of coherence, which is a multi-temporal change detection. The index, named Temporal Stability Index (TSI), has been tested to estimate the dune movements in the desert of the United Arab Emirates and Egypt.

The paper is structured as follows. In Section 2 the adopted methodology for computation of TSI is illustrated. Section 3 provides information about the use cases areas, the processed SAR imagery, and the obtained results. In the last section, conclusions are reported.

\section{METHODOLOGY}

This section presents the complete procedure developed for the analysis of SAR-derived scene stability from a stack of Sentinel1 imagery (Figure 1). In the following, the pre-processing steps aimed at preparing data for the interferometric process, and the processing steps to compute the coherence-based TSI are proposed.

\footnotetext{
* Corresponding author
} 
It is worth noting that most of the proposed procedures have been performed by means of SNAP (Sentinel Application Platform), the free open source toolbox developed by ESA to support the scientific exploitation of Sentinel missions.

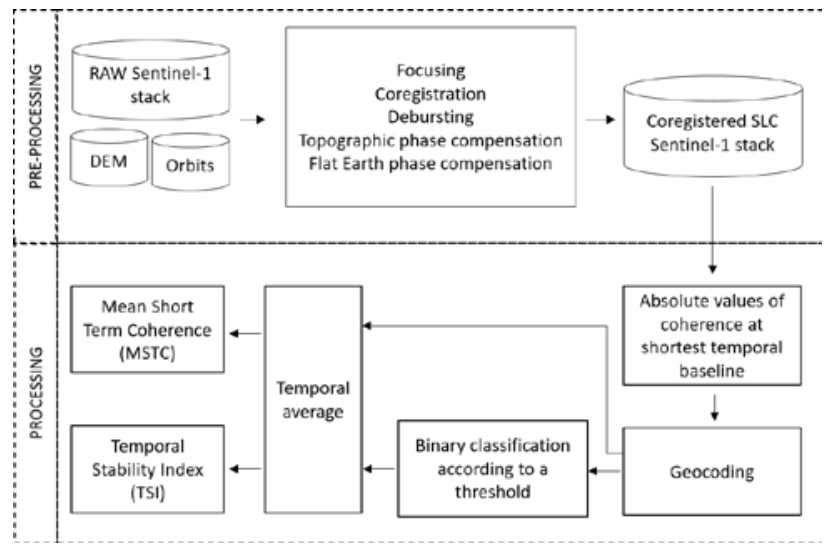

Figure 1. Scheme of the complete procedure to obtain the Temporal Stability Index from a stack of Sentinel-1 imagery.

\subsection{Pre-processing}

The input of the pre-processing procedure, proposed in Figure 1, is usually a stack of Level-1 Single Look Complex (SLC) images. However, if the stack is based on a huge number of images, Level-0 RAW images are often preferred because of their smaller size which makes downloading faster. In this case, a focusing phase is required to convert Level-0 products into Level-1 SLC.

Once the SLC dataset is available, the next step of the preprocessing is the coregistration, which allows to align all the images to a selected reference one with sub-pixel accuracy (Sansosti et al., 2006). Since Sentinel-1 is characterized by burstmode acquisitions, a proper debursting is needed to join all bursts into a single image (De Zan, Monti Guarnieri, 2006).

As the proposed change detection analysis is based on coherent technique, thus exploits both the amplitude and the phase, the interferometric processing needs to be applied to perform a proper calibration of the phases (Freeman, 1992).

The InSAR technique (Ferretti et al., 2007) estimates terrain changes by exploiting the phase difference between two coregistered complex radar observations acquired in the same area at different times (Figure 2). As the phase senses the radartarget distance, a variation of the phase reflects the variation of the radar-target distance. This variation between observations can be attributed to very few contributions:

- the possible displacement of the target;

- the local topography effect due to the difference in sensor trajectory during the acquisitions;

- the so-called flat Earth effect due to the Earth curvature;

- changes in atmosphere conditions (humidity, temperature, etc.).

As the present work is interested in detecting the contribution only related to possible displacements of the target, all the other contributions need to be removed.
The removal of topographic and flat Earth phases is performed using an existing Digital Elevation Model (DEM), like the Shuttle Radar Topography Mission ${ }^{1}$ (SRTM).

Regarding the removal of effects due to changes in atmospheric conditions, well-established methods are proposed in the literature (Gomba et al., 2017; Monti Guarnieri et al., 2018; Manzoni et al., 2020). However, the effects are significant only if global scale phenomena are considered. Since the present work deals with local-scale analysis, the atmospheric phase compensation was not considered.

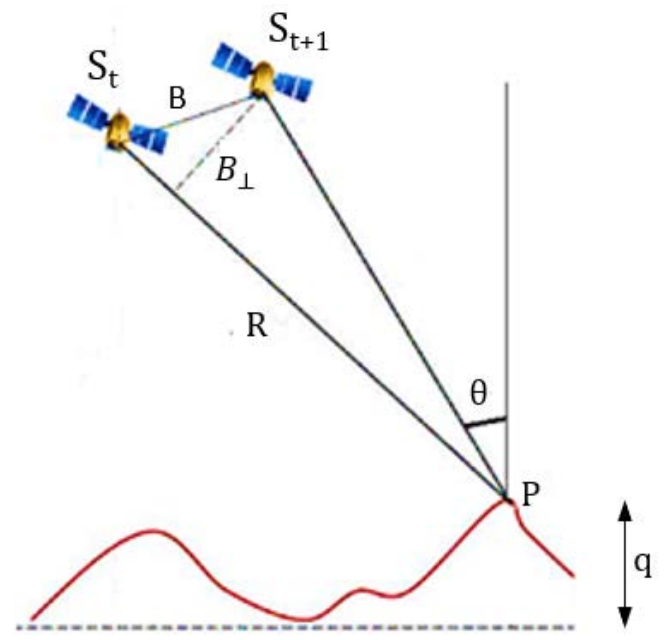

Figure 2. Geometry of an interferometric SAR system. The target $\mathrm{P}$ is imaged by radar $\mathrm{S}$ at different times.

\subsection{Processing}

The coregistered SLC stack without topographic and flat Earth phase contribution represents the input for the computation of the coherence and the TSI.

2.2.1 Coherence: Coherence is the linear correlation coefficient between two complex SLC images. Given the two observations, $x_{m}$ and $x_{n}$, of the same target located in $P$, the coherence is estimated using a spatial averaging as proposed in Equation 1:

$$
\hat{\gamma}(P)=\frac{\sum_{i} \sum_{j} x_{n}\left(P_{i}, P_{j}\right) x_{m}^{*}\left(P_{i}, P_{j}\right)}{\sqrt{\sum_{i} \sum_{j}\left|x_{n}\left(P_{i}, P_{j}\right)\right|^{2} \sum_{i} \sum_{j}\left|x_{m}\left(P_{i}, P_{j}\right)\right|^{2}}}
$$

where * indicates the complex-conjugate while $i, j$ represents the row and column indices of the spatial estimation windows.

The absolute value of the coherence, $|\gamma(\mathrm{P})|$, which ranges in the interval $[0,1]$, is an indicator of the stability of the scene. If $|\gamma(\mathrm{P})|$ $=1$ the target remains stable (i.e. the two observations are linearly dependent); $|\gamma(\mathrm{P})|=0$ indicates that the two observations of the target are independent, while if $0<|\gamma(\mathrm{P})|<1$ some dependence between the observations can be observed.

\footnotetext{
${ }^{1}$ https://www2.jpl.nasa.gov/srtm/
} 
If a stack of multi-temporal coregistered SLC images is available, useful information about the scene can be provided by the mean of short-term coherence (MSTC) index, which can be computed as proposed in Figure 1. The first step consists in calculating the coherence for each pair of SLC images acquired at the shortest temporal baseline and then take the absolute value. After that, the images are geocoded, i.e. they are converted from the slant range geometry typical of SAR imagery into a map coordinate system (Small, Schubert, 2008). Finally, the temporal average of the geocoded images is performed.

2.2.2 Temporal Stability Index (TSI): The TSI we are proposing is directly derived from the coherence to have a measure of the fraction of time in which a target remains stable. Its computation is performed through a very simple and fast procedure, which differs from the MSTC computation procedure just for an additional step (Figure 1).

In fact, given the stack of geocoded absolute values of coherence computed between the SLC pairs of images at the shortest temporal baseline, a classification step is introduced to exclude the totally uncorrelated samples. The identification of these samples is performed according to a specific threshold $\gamma$ that corresponds to the noise and can be computed a priori as suggested by Ferretti et al. (2007). Once $\gamma$ is known, each absolute value of the coherence image is classified by assigning value 1 (i.e. stability) to pixels with values greater than $\gamma$, and value 0 (i.e. instability) to the remaining pixels. Thus, at the end of this step binary images are generated.

The last step involves the temporal averaging of the binary images previously computed to obtain the final TSI, which is characterized by pixel values ranging in the interval $[0,1]$ :

- $\quad$ TSI equal to 1 means that a target remained stable $N$ out of $N$ images;

- TSI less than 1 points out the presence of some instability occurred in some epochs during the overall time-span of the stack. Values of TSI very close to 0 indicates that the target has been found stable only for a very limited period;

- $\quad$ TSI $=0$ means that the target was never found stable, or -equivalently- that is pure noise.

\section{USE CASES}

The TSI index has been computed on the desertic areas of Egypt and the United Arab Emirates to evaluate the instability due to dunes and sand sheets movements. Within this section, information about the use cases areas, the processed SAR imagery stacks, and the obtained results are presented.

\subsection{Data}

The use cases regions of interest (ROIs), identified by red boxes in Figure 3, were selected by considering the presence of transportation infrastructure, urban and agricultural lands which can be threatened by the movement of the dunes. Sentinel-1 ensures the full coverage of both Egypt and the United Arab Emirates: the imagery stacks were selected by considering the orbits able to guarantees the maximum coverage of the ROIs.

The period considered for the study is 2015-2018. The total number of Level-0 images acquired by Sentinel-1A and Sentinel$1 \mathrm{~B}$ within this temporal interval is 65 for the United Arab Emirates use case and 74 for Egypt one. While the products available for years 2017 and 2018 are characterized by a temporal baseline of 12 days, few products are instead available for years 2015 (the year of Sentinel-1A launch) and 2016. More information about the collected images are reported in Table 1.
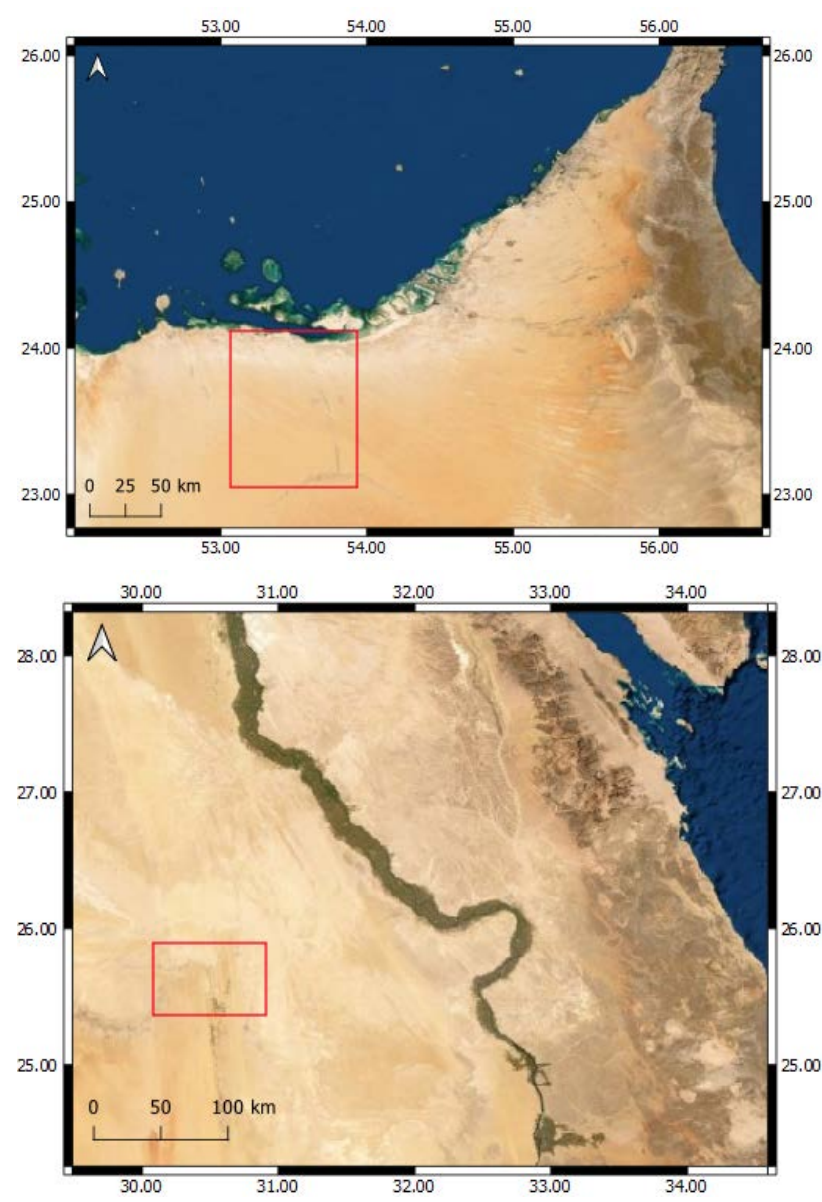

Figure 3. Regions of interest (red boxes) selected for the United Arab Emirates (top) and Egypt (bottom) use cases (ㄷ ESRI satellite Copyright). Coordinates are expressed in WGS84.

\begin{tabular}{|l|c|c|}
\hline & $\begin{array}{c}\text { United Arab } \\
\text { Emirates }\end{array}$ & Egypt \\
\hline Sensor & S1A & S1A-S1B \\
Relative Orbit & 28 & 131 \\
Pass & Ascending & Ascending \\
Number of images & 65 & 74 \\
First image acquisition date & $2015-04-29$ & $2015-05-06$ \\
Last image acquisition date & $2018-12-21$ & $2018-12-22$ \\
Centre coordinate (Lat) & 23.7069 & 25.284 \\
Centre coordinate (Long) & 53.3529 & 30.242 \\
\hline
\end{tabular}

Table 1. Information about SAR imagery collected for the United Arab Emirates and Egypt use cases.

\subsection{Results and discussion}

The procedure described in section 2 was applied to the imagery stack of each use case, and coherence and TSI maps were computed. In the following, the main results are showed and discussed. 
3.2.1 United Arab Emirates: The coherence images for the United Arab Emirates were obtained by applying a spatial estimation window of $120 \mathrm{~m}$ in range and $170 \mathrm{~m}$ in azimuth. All the images were processed at the $2 \times$ upsampled pixel spacing of $2.5 \mathrm{~m}$ in range $\times 12.5$ in azimuth, and - after processing geocoded into a $20 \mathrm{~m} \times 20 \mathrm{~m}$ geographical grid.

Figure 4a presents the absolute values of the United Arab Emirates coherence computed between the two images at the longest temporal baseline (i.e. April 2015 - December 2018). The result highlights low coherence (dark spots), thus high decorrelation between the two considered images. The few areas characterized by high coherence values (bright spots) correspond to build areas or, in general, manmade objects.

To obtain the MSTC map, proposed in Figure 4b, a coherence map was generated for each pair of coregistered SLC images characterized by the shortest temporal baseline. A total of 64 coherence images were obtained and the mean of their absolute values was computed. The resulting map highlights with detailed colour gradation the areas characterized by high coherence (green-blue colour), where stable structures are present; these areas, corresponding to transport infrastructures and urban areas/oasis, are mainly located in the North (coastal region), in the North-East (Zayed city), and in the South-East (Liwa oasis). The low coherence areas, which represent most of the region of interest, are instead characterized by a uniform red colour indicating instability; here, the index gives very poor information. Notice that - being an average - the MSTC index is not capable to distinguish between a target that is highly stable but only for a limited period of time, and one that is moderately stable for all times.

Therefore, the two coherence maps do not provide indication on non-stationary intermittent instability, like those occurring to the desert areas under study. In fact, the dune movement involves high-frequency changes over time of the distributed target (i.e. the dunes) due to seasonal variation of wind direction and speed. A better understanding of the phenomenon can be obtained by getting information related to the fraction of time the target remained stable in the four years. To this aim, the TSI was conceived.
The TSI map of the United Arab Emirates is shown in Figure 5. It is worth noting that the no colour in the ROI indicates TSI $=0$, thus total instability. Compared to the MSTC (Figure 4b), this map presents less detail (i.e. less colour graduation) in high coherence areas and more detail in low coherence areas. This is the desired effect to enhance the difference between totally unstable and partially unstable areas.

Since the use case area is characterized by heterogeneous structures, a further investigation at a local scale was performed to identify different TSI behaviours. In particular, the analysis was focused on the four sub-areas highlighted in Figure 5:

- the analysis of sub-area $a$ (Figure 5a) highlights very stable areas characterized by TSI equal to 1 (blue colour) which are similar in shape to dunes; however, the Google satellite image referred to 2019 seems to indicate that these areas are rock. The surrounding terrain is characterized by red colour or no colour, which indicates high or total instability due to continuous sand sheets movements.;

- the sub-area $b$ (Figure $5 b$ ) is characterized by very high instability over the considered years. This behaviour is confirmed by Google satellite image which shows that sand is covering the forest areas close to the road suggesting sand sheet movements in the region;

- the sub-area $c$ (Figure 5c) shows few stable structures, such as roads and buildings, surrounded by totally unstable areas. Here, sand has a constant of movement much lower than 12 days (temporal baseline);

- The sub-area $d$ (Figure $5 \mathrm{~d}$ ) is characterized by high TSI values, thus high stability. Some structures, like roads, are easily recognizable.

It is clear that the information about the stability of the scene in time and space obtained through the analysis of the TSI index can represent a valuable resource for the adoption of mitigation risk measures and the conscious planning of infrastructure construction. (a)

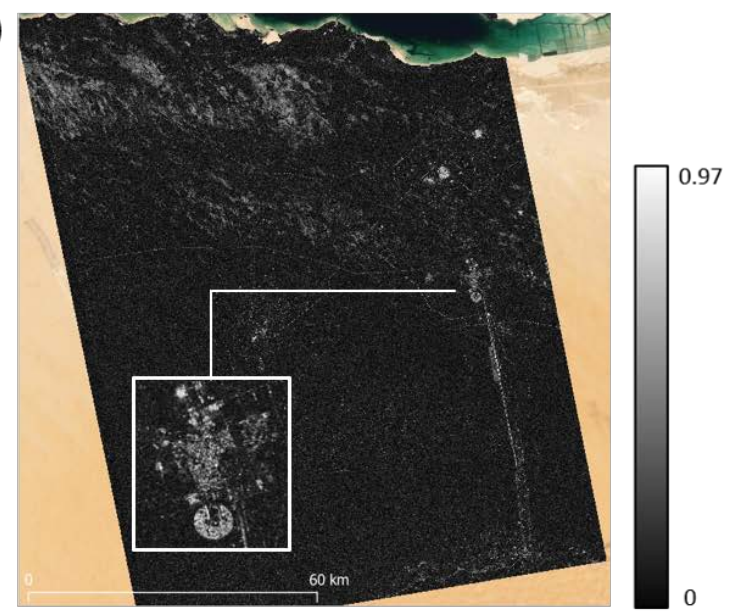

(b)

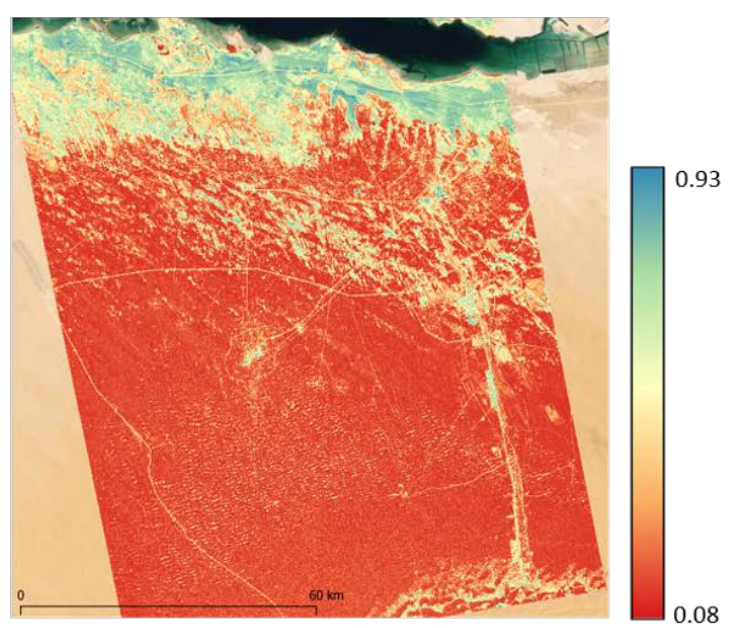

Figure 4. The United Arab Emirates use case: (a) absolute value of the coherence computed between the two images at the longest temporal baseline; the higher the coherence absolute values, the higher the stability of the scene; (b) mean of short-term coherence (MSTC); (C ESRI satellite Copyright). 


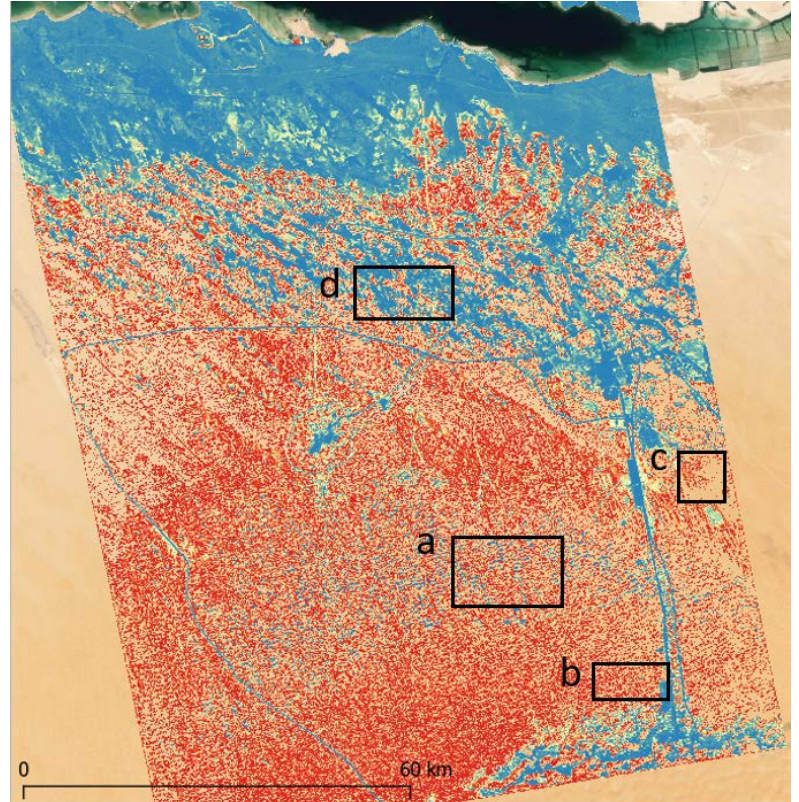

(a)
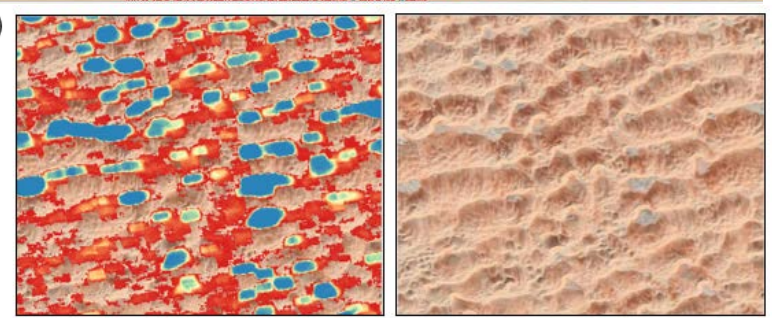

(b)
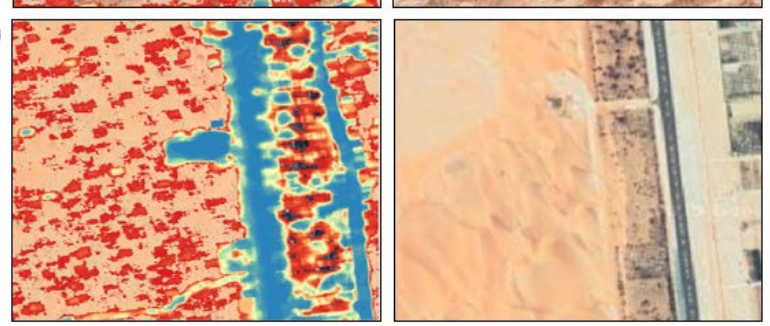

(c)

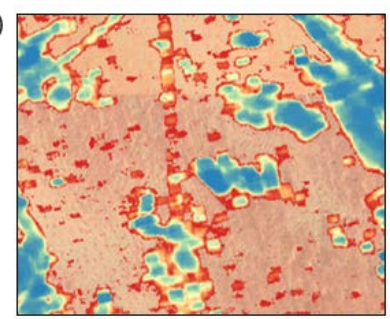

(d)
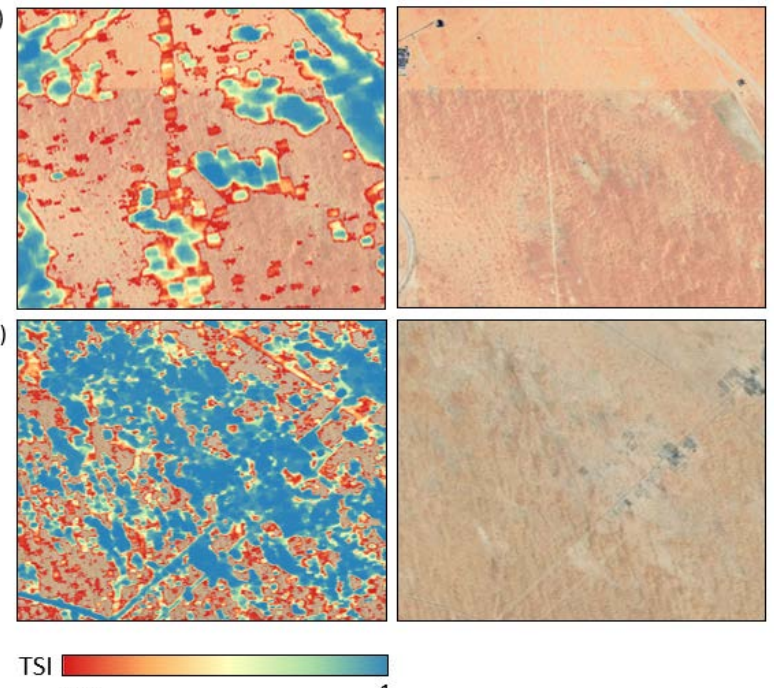

0.01

Figure 5. Temporal Stability Index (TSI) computed for the

United Arab Emirates use case. At the top, the map related to the whole region of interest is proposed (C ESRI satellite

Copyright). For each sub-area highlighted in the Figure, the TSI and the Google satellite optical image is presented (@ Google 2019 Copyright).
3.2.2 Egypt: The coherence images for Egypt have been generated by applying a spatial estimation window of $45 \mathrm{~m}$ in range and $65 \mathrm{~m}$ in azimuth. As the United Arab Emirates use case, all the images were processed at the $2 \times$ upsampled pixel spacing of $2.5 \mathrm{~m}$ in range $\times 12.5$ in azimuth, and - after processing - geocoded into a $20 \mathrm{~m} \times 20 \mathrm{~m}$ geographical grid.

First, the coherence absolute value map between the SLC images characterized by the longest temporal baseline (May 2015 - December 2018) was generated. The resulting map, proposed in Figure 6a, generally shows high coherence absolute values, meaning that no significant changes occurred to the finescale texture, at the sub-centimeter level. Thus, results exclude dune motions and other macroscopic changes in morphology. Only a region in correspondence of El-Kharga Oasis shows possible movements/changes (dark spots, i.e. low coherence).

Figure 6c presents the MSTC index computed over the area of interest by averaging the absolute value of 73 coherence maps. The map shows high coherence (green/blue colours) in most of the investigated area suggesting that high stability characterized the area over the considered years. However, the region presents also structures at low coherence that can be easily identified as dunes. Decorrelation is due to the combination of the continuous dune displacements, and penetration of electromagnetic (EM) wave in the sand, up to $50 \mathrm{~cm}$, that causes a weak backscatter, very close to the noise floor. The two conditions are correlated, as a thick sand layer is subject to be shifted by winds, and each of the two is a potential decorrelation loss. As a result, low coherence is here a proxy for a sandy dune, although not necessarily.

The TSI (Figure 6b) confirms its capability of better identifying, compared to MSTC, instabilities. In particular, the El-Kharga Oasis region clearly shows the presence of very unstable structures characterized by continuous movements that can be easily recognized as barchan (crescentic) and linear dunes. The obtained results are confirmed by the literature: Hererer (2010) exploited satellite optical images to analyzed the dunes behavior in the El-Kharga Depression area by estimating a dune advance ranging from $55 \mathrm{~m}$ to $128 \mathrm{~m}$ between 1987 and 2000. Hossoup (2019) confirms that El-Kharga Oasis is suffering from the dunes motion natural hazard and indicates a rate of movement of crescentic dunes which varies from 0.5 to 14 meters per year.

A qualitative comparison between SAR-derived information and evidence detectable from optical satellite images is finally presented. Figure $6 \mathrm{~d}$ shows a satellite optical image acquired by the Sentinel-2 mission in June 2018; to enhance the visualization of sand (light blue color), an adaptive histogram equalization was performed on the bands more sensitive to dunes.

The focus of the El-Kharga Oasis area shows that both the two approaches identify the presence of dunes, intended as the accumulation of sand subject to instability due to wind effect; however, the optical system senses thin layers of sand-sheets that are totally transparent to radar. This is evident by observing the wakes that are quite similar to the dunes themselves in optical, and quite well distinguished in the TSI. The discrepancies can be explained by considering the different observations that characterize the two approaches: the optical systems provide quite good sensitivity to discriminate sand by its "colour", disregarding the thickness, while the radar senses the sand by its penetration depth (up to $50 \mathrm{~cm}$ ) or by its instability. 
(a)

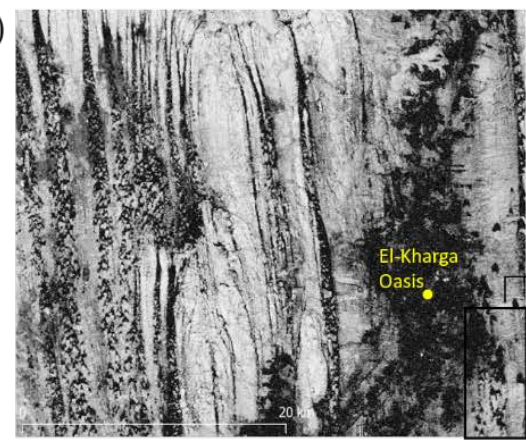

(b)

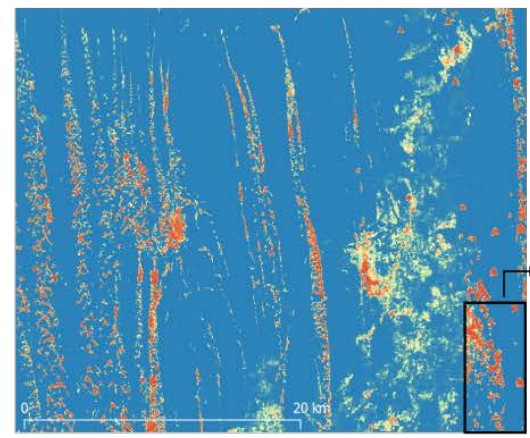

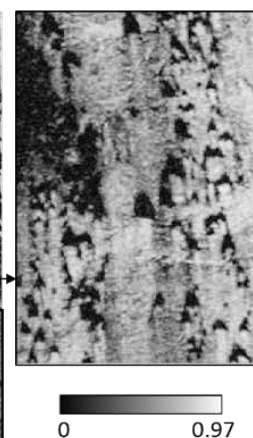

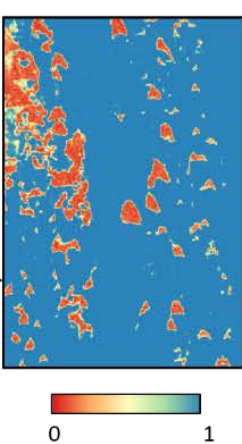

(c)
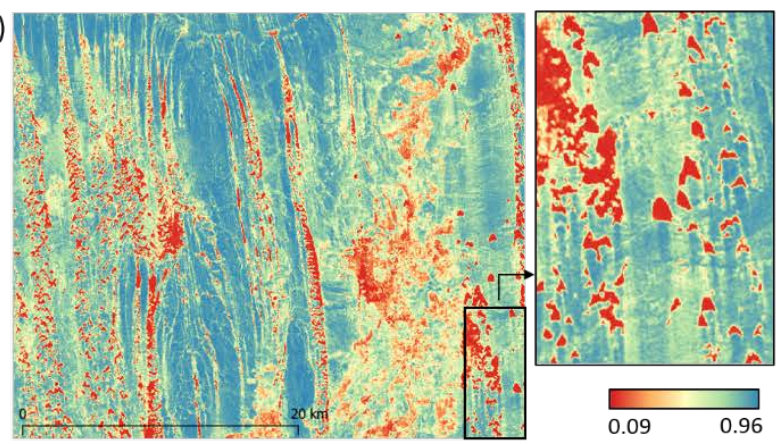

(d)
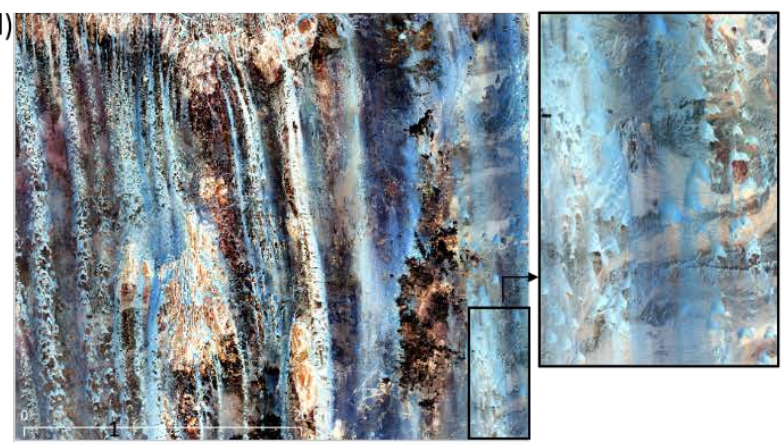

Figure 6. Egypt use case: (a) coherence absolute values computed between the two images at the longest temporal baseline; (b) Temporal Stability Index; (c) mean of short-term coherence (MSTC); (d) Sentinel-2 optical image after an adaptive histogram equalization which enhances sand visualization (light blue colour).

\section{CONCLUSION}

In this investigation, we propose a novel, coherence-based index, named Temporal Stability Index (TSI), for the evaluation in time and space of SAR-derived scene stability. The index is suited to characterize non-stationary stability, particularly to assess the percentage of time a target remains stable over time.

The TSI was computed for the evaluation of instability in desertic areas and showed interesting results. In particular, the TSI analysis in the United Arab Emirates allowed identifying subareas characterized by very different stability behaviours; this is crucial information for the implementation of risk mitigation measures and the strategic planning of infrastructure construction.

Moreover, the Egypt use case proved the TSI capability to identify dunes structures; in fact, the index identifies dunes as they decorrelate because of changes due to movement, penetration in the volume, and very low backscatter.

The TSI was able to provide in a very simple way a measure of the temporal behaviour of dunes structures and, more generally, its use can be extended to understand the stability/instability over time of whatever target characterized by high-frequency changes.

\section{ACKNOWLEDGEMENTS}

The authors would like to thank Diego Frizzi and Stefano Martinati of Saipem S.p.A. for their active contribution to the research in the framework of the project "Satellite analysis of desert sand dunes migration”.

\section{REFERENCES}

Dabboor, M., White, L., Brisco, B., Charbonneau, F., 2015. Change Detection with Compact Polarimetric SAR for Monitoring Wetlands. Canadian Journal of Remote Sensing, 41(5), 408-417.

De Zan, F., Monti Guarnieri, A., 2006. TOPSAR: Terrain Observation by Progressive Scans. IEEE Transactions on Geoscience and Remote Sensing, 44 (9), 2352-2360.

Durieux, A.M.S., Calef, M.T., Arko, S., Chartrand, R., Kontgis, C., Keisler, R., Warren, M.S., 2019. Monitoring forest disturbance using change detection on synthetic aperture radar imagery. Proc. SPIE 11139, Applications of Machine Learning, 1113916.

Engeset, R.V., Kohler, J., Melvold, K., Lundén, B., 2002. Change detection and monitoring of glacier mass balance and facies using ERS SAR winter images over Svalbard. International Journal of Remote Sensing, 23(10), 2023-2050.

Ferretti, A., Monti-Guarnieri, A., Prati, C., Rocca, F., Massonnet, D., 2007. InSAR Principles: Guidelines for SAR Interferometry Processing and Interpretation. TM-19. ESA Publications, The Netherlands.

Freeman, A., 1992. SAR calibration: an overview. IEEE Transactions on Geoscience and Remote Sensing, 30(6), 11071121.

Gomba, G., González, F. R., De Zan, F., 2017. Ionospheric phase screen compensation for the Sentinel-1 TOPS and ALOS-2 ScanSAR modes. IEEE Trans. Geosci. Remote Sens., 55(1), 223235. 
Hassoup, A., 2019. Sand dunes hazard assessment in El-Kharga Oasis, Egypt. Geophysical Research Abstracts, 21, EGU20191714.

Hereher, M.E., 2010. Sand movement patterns in the Western Desert of Egypt: an environmental concern. Environ Earth Sci., 59, 1119-1127.

Jung, J., Kim, D.-J., Lavalle, M., Yun, S.-H., 2016. Coherent change detection using InSAR temporal decorrelation model: A case study for volcanic ash detection. IEEE Trans. Geosci. Remote Sens., 54(10), 5765-5775.

Jung, J., Kim, D.-J., Yun, S.-H., Lavalle, M., 2017. Damage mapping based on coherence model using multi-temporal polarimetric-interferometric UAVSAR data. Proc. IEEE Int. Geosci. Remote Sens. Symp. (IGARSS), 189-192.

Manzoni, M., Monti-Guarnieri, A.V., Realini, E., Venuti, G., 2020. Joint Exploitation of SAR and GNSS for Atmospheric Phase Screens Retrieval Aimed at Numerical Weather Prediction Model Ingestion. Remote Sens., 12, 654.

Monti Guarnieri, A., Leanza, A., Recchia, A., Tebaldini, S., Venuti, G., 2018. Atmospheric Phase Screen in GEO-SAR: Estimation and Compensation. IEEE Transactions on Geoscience and Remote Sensing, 56(3), 1668-1679.

Monti-Guarnieri, A.V., Brovelli, M.A., Manzoni, M., Mariotti d'Alessandro, M., Molinari, M.E., Oxoli, D., 2018. Coherent Change Detection for Multipass SAR. IEEE Transactions on Geoscience and Remote Sensing, 56(11), 6811-6822.

Preiss, M., Gray, D. A., Stacy, N. J. S., 2006. Detecting scene changes using synthetic aperture radar interferometry. IEEE Trans. Geosci. Remote Sens., 44(8), 2041-2054.

Rignot, E. J. M., van Zyl, J. J., 1993. Change detection techniques for ERS-1 SAR data. IEEE Trans. Geosci. Remote Sens., 31(4), 896-906.

Sansosti, E., Berardino, P., Manunta, M., Serafino, F., Fornaro, G., 2006. Geometrical SAR image registration. IEEE Trans. Geosci. Remote Sens., 44, 2861-2870.

Small, D., Schubert, A., 2008. Guide to ASAR Geocoding. Report RSL-ASAR-GC-AD. Remote Sensing Laboratories, University of Zurich, Zurich, Switzerland.

Tamura, M., 2015. Mapping urban damage of the 2011 EastJapan earthquake using multi-temporal PALSAR images. Proc. IEEE Int. Geosci. Remote Sens. Symp. (IGARSS), 3576-3578. 